\title{
A Review of key technologies in flight simulator
}

\author{
Siyi Liu ${ }^{1, a}$ \\ ${ }^{1}$ School of Mechanical Engineering, Jincheng College of Sichuan University, \\ Chengdu, Sichuan 611731, China. \\ àichaelia1996@qq.com
}

Keywords: Flight simulator, flight control system, flight simulation, aerodynamic modeling.

\begin{abstract}
Flight simulator is the key equipment for aircraft design research and pilot training. Flight simulation technology is irreplaceable in the development and production of aircraft. Flight simulation technology involves the field of aircraft structure, air independence, flight control and flight power. This paper summaries the modeling and simulation techniques of aircraft movement, aircraft control system and flight environment, and provides feasible analysis of future flight simulation development trend.
\end{abstract}

\section{Introduction}

Flight simulation system is an important tool for modern aircraft design and development. It can support dynamic verification of aircraft design. Flight simulation system simulates the full-envelope characteristics of the ground and air of the aircraft by establishing models such as aerodynamic, ground characteristics, landing gear, mass, motion equation, and atmospheric environment. Key technologies of aircraft simulation include aircraft motion simulation technology, flight control simulation technology, aircraft avionics system simulation technology and flight environment simulation technology.

Flight simulator can be divided into engineering flight simulator and training flight simulator. The engineering flight simulator has a high degree of fidelity and a certain degree of versatility. It can be used in ground assessment and tests of aircraft characteristics and airborne equipment, so as to prompt the design, improve the performance, figure out the existing problems in aircraft development phase and reduce expenses. The cockpit of training flight simulator is exactly the same as that of the real aircraft. Through a variety of physical simulation equipment, it provides the pilot with the similar or even the same feeling of real flights. It has become both an indispensable part of the pilot training process and a key tool for air crash analysis.

According to the development of simulation technology, aircraft simulation has gone through five stages: physical simulation, simulator simulation, digital computer simulation, 3D visual multimedia interactive simulation based on graphics workstations and virtual environment (VE) and virtual reality (VR). The whole digital flight simulation system consists of two parts; one part is simulating the flight equation, the rudder deflection, the weight change and other system parameter change information of the aircraft itself. The other part is simulating the plane's coordinate position, atmospheric condition, and radio station signals, meteorological information detected by weather radar, terrain information, GPS signals, air traffic control information and navigation information.

\section{Modeling and simulation technology of aircrafts}

The flight state parameters include: Euler angle (roll angle, yaw angle, pitch angle), angle of attack, side slip angle, track deviation angle, track inclination angle and so on. After determining these angles, aircraft movement state can be obtained. It is necessary to analyse the various forces and moments of the aircraft. Then, the six-degree-of-freedom nonlinear full-motion differential equation 
is used to combine the vehicle force and the moment equation to solve the motion parameters and state parameters of the aircraft.

\subsection{Requirements of aircraft modelling and simulation.}

For conventional aircrafts, we should establish a six-degree-of-freedom nonlinear full-motion differential equation set. Real-time numerical integration of the equation set helps to obtain the state of the aircraft at each moment. The impact of aerodynamic elasticity is needed to consider for large aircraft. Elastic degrees of freedom can be added to the kinetic equation. In order to improve the fidelity of flight simulation, the aerodynamic model under the Earth's curvature and the ellipsoidal coordinate system should be taken consideration, so that global flight is simulated.

\subsection{Aircraft kinematics and dynamics equations.}

Aerodynamic model describes aerodynamic characteristics under various conditions. Aerodynamic forces and moments are functions of flight condition parameters. By applying linearly aerodynamic theory, aerodynamic forces and moments are decomposed of a number of stability derivatives related to flight status, i.e., aerodynamic derivatives. The establishment of aerodynamic force and moment models is usually based on the combination of deduction and induction. Aerodynamic derivatives of aerodynamic coefficients, such as resistance, side force, lift, roll moment, pitch moment, yaw moment are decided according to the principle of flight mechanics. Aerodynamic derivatives are usually calculated by wind tunnel test, flight test and computational fluid dynamics.

\subsection{Atmospheric environment modelling.}

There are a variety of atmospheric disturbances in real atmosphere, which have a very large impact on the quality of the aircraft. In order to increase the authenticity of flight simulation, it is necessary to establish an atmospheric disturbance model similar to the real atmosphere. Atmospheric disturbances include many forms: low-level steady-state winds, atmospheric turbulence, and gusts. Atmospheric turbulence is the most complex, so the model is most difficult to establish. The formation and appearance of atmospheric turbulence are generally related to terrain induction, heat exchange, wind shear and other factors. At the same time, towed trails of aircrafts and ships will also form turbulent areas. For example, for the movement of ships, turbulent areas will also form at the ships' tail areas, which are known as the ship streams. They form because of the wind flow through a variety of buildings on decks, which greatly impacts the carrier-based aircraft landing.

\section{Flight control system modeling and simulation technology}

The flight control system controls the rudder movement and the engine thrust, so that the aircraft can fly to a certain attitude along the scheduled flight path. Flight control system directly affects flight safety. In order to improve the flight quality and reduce the wear of the aircraft operating system components, the modern civil aircraft put forward higher requirements for the flight control system, thus effectively improving the reliability and safety of the aircraft. The Flight control system's performance directly affects the flight safety and aircraft performance as one of the core systems. The physical system is analysed and researched by establishing a high-fidelity flight control system model.

\subsection{Flight control system modeling and simulation requirements.}

The flight control system used in the flight simulator not only simulates the basic states of the aircraft, such as airspeed, altitude, heading, etc., but also simulates the abilities of the aircraft, such as climbing or descending speed control, co-turning and automatic landing. Through the discussion and research of the flight control system, the typical link model of the flight control system is established. The model configuration is carried out according to the design requirements, and a complete flight control system modelling process is summarized.

\subsection{Composition and working principle of automatic flight control system.}

According to different sources of control systems, they can be classified as manual control systems and automatic flight control systems. The manual control system is mainly controlled by the driver to achieve the control of the aircraft rudder surface deflection, in order to achieve the attitude of the aircraft changes, height changes and other actions. The automatic flight control system is a system 
that replacing the pilot to operate the aircraft by the control system, including the longitudinal control system, the horizontal heading control system, the automatic landing system, the automatic throttle system and so on. Flight control system generally refers to the automatic flight control system. It is mainly used to achieve flight stability and control tasks.

Flight control system modelling is usually modular and hierarchical. According to physical functions of the system, the flight control system is divided into various sub-modules. Each sub-module uses object-oriented technology to achieve the module's packaging and inheritance. In order to establish the flight control system model accurately, supports of the atmospheric environment, flight management system and engine system are required. In order to establish a real model of high fidelity, aircraft pneumatic data packets, flight control packets, engine data packets are necessary. Moreover, the Identification criteria of training- type flight simulator can be used as a reference for measuring model fidelity.

\section{Flight environment modeling and simulation technology}

\subsection{Dynamic simulation.}

Due to limited strokes of the hydraulic cylinder, it must be reversed to the neutral position after the completion of an action so that the continuous motion simulation can have sufficient strokes. Usually this additional motion that turning back to the neutral position is called the Washout of the movement. Because the washout is an additional movement, it is needed to not feel the existence of the movement for pilots, which requires the movement should be completed at an almost uniform speed and acceleration less than the human feeling threshold (approximately 0.02g).[Since the washout is an additional action, it should be taken without feeling its existence, which should be completed at an almost standardised speed with an acceleration that is less than the human felling threshold (approximately 0.02g).] Thus suppressing jamming jitter and improving motion smoothness are main design goals for the flight simulator motion system. The key to dynamic simulation is the washout algorithm. The effect of the washout algorithm is simulating the movement sensation in the infinite motion space by using the limited motion space of the six degrees of freedom parallel mechanism. At present, the most widely used algorithm is the adaptive washout algorithm. The adaptive washout algorithm can adjust the adaptive parameters according to the current position of the motion platform so that it can provide a high fidelity motion feeling near the neutral position.

The movement sensation is an important channel for the pilot to judge the flight status, so the fidelity of the dynamic simulation directly affects the fidelity of the flight simulator. The six-degree-of-freedom parallel mechanism provides a sense of movement for the flight simulator. It can provide the instantaneous overload dynamic feeling, persistent feeling of gravity component and partial jitter impact information for pilot. It is a transient overload simulation equipment controlled by a real-time digital computer and can provide freedom degrees of pitch, roll, yaw, lift, vertical translation and lateral translation. Therefore, the performance merits of the motion system are directly related to the flight simulation fidelity. The research on the mechanics theory, key components and control strategies of the parallel 6-DOF motion system is the key technology to optimise the design of the motion system and to improve the performance of the flight simulator. This is the most basic task of the six-degree-of-freedom motion platform motion analysis. It is also the basis for the speed, acceleration, error analysis, workspace analysis, dynamic mechanics and institutional synthesis. In contrast to the series mechanism, the position of the parallel mechanism is relatively simple. The positive solution of the position is multi-solution because of the nonlinear relationship between the platform position and the expansion and contraction amount of each branch, which is more difficult to solve and is one of the hot issues.

\subsection{Visual simulation.}

The flight simulator and its visual system have developed sharply in recent years. Related support technologies such as optical imaging technology, nonlinear distortion correction technology, rapid modelling technology, computer graphics acceleration rendering technology, virtual environment effects simulation, realistic atmospheric environment simulation, interactivity testing, collision 
detection technology and 3D terrain visualisation technology are hot spots in researches. Compared with other visual systems, flight simulator vision system has its own characteristics:

(1)Real-time displaying of real three-dimensional scenes related to simulator gestures and scene settings, including airport scenes and a wide range of terrain; (2)Simulating visual environments required for flight, including atmospheric environments such as precipitation, clouds and the environment Fog, etc., as well as the simulation of the airport and the ambient light; (3) The visual system should respond to the control instructions of the coaching station; (4) The visual system can meet the terrain height inquiry and the collision detection processing; (5)Updating rate is not less than 20 frames / sec, and try to ensure that the frame rate is constant; (6)The system horizontal field angle is not less than 150 degrees, the vertical field view angle is not less than 30 degrees; (7) The system response delay should be under 150 milliseconds at which the aircraft begins to respond.

\subsection{Sound simulation.}

The sound simulation system is an important part of the flight simulator, which provides pilots with immersive sound effects to help to correctly determine the state of the aircraft. With the continuous development of computer technology, sound simulation software development has made great progress. Modular, integrating and portability is getting higher and higher. However, for different types of aircrafts, development of sound simulation system needs to mix the sound source from the original recording, making the sound source extraction workload huge.

At home and abroad, the mainly used sound analysis and synthesis technology is Fourier analysis and synthesis methodology. The time domain signal is converted into a frequency domain signal by Fourier transform and a variety of processes are conducted in the frequency domain. Problems that are hard to solve in the time domain can be easily solved in the frequency domain. Finally through the inverse Fourier transform, the signal from the frequency domain is converted back to the time domain. It is not only used in the field of sound, but also widely used in other fields.

\section{Development and challenges of flight simulation}

This paper introduces the key technologies of aircraft simulation, including motion modelling and simulation, flight control system modelling and simulation, and flight environment modelling simulation. When analysing the aircraft modelling and simulation, the requirements of motion modelling and simulation, aircraft kinematics and dynamics equations are mentioned, and the atmospheric environment modelling is also discussed. In the aspect of aircraft control system modelling and simulation technology, it introduces the composition and working principle of automatic flight control system. As for flight environment modelling and simulation technology, dynamic simulation, visual simulation and sound simulation and relevant issues are discussed.

In the future, simulation technology research will focus on large-scale complex system simulation. The main features are fuzzy and intelligent. The software will form an integrated modelling and simulation environment with powerful, complete information and structure by supporting the object-oriented simulation development language, general or special simulation software package. Six degrees of freedom movement system is the key of the simulation system. The motion smoothness is a crucial research direction of the future simulation technology. The virtual reality training simulator will also replace that based on the traditional technology.

\section{References}

[1] XING Yong-gang, TANG Shuo. Modeling and Simulation of Propulsion System for Hypersonic Cruise Vehicle[J].COMPUTER SIMULATION,2011,28(11):54-58.

[2] WANG Hua-dong. Modeling and simulation of atmospheric turbulence[J]. FLIGHT DYNAMICS,2014,32(1):84-87

[3] TAN Zhen-zhen. Research on Modeling and Simulation for Flight Control System of Large of Large Civil Aircraft[D].Nanjing University of Aeronautics and Astronautics,2014. 
[4] TONG Zhong-xiang, WANG Xiao-dong. Development and Prospect of Flight Simulation Technology[J]. FLIGHT DYNAMICS,2002,20(3):5-8.

[5] DENG Chang-hao. Application of Flight Simulation Technology in Civil Aviation System Test [C]// Chinese Society of Aeronautics and Astronautics.2006

[6] QI LIN,LANG Bin, CHENG Nong. Real-time Simulation of Flight Management System[J] .Journal of System Simulation,2009,20(8):332-336.

[7] YANG Yu. Research on Key Technology of Motion Cueing for Flight Simulator[D].Harbin Institute of Technology,2010.

[8] ZHANG Lei. Flight Simulation Modeling and Software Development for Flight Simulator [D]. Harbin Institute of Technology,2009.

[9] LV Ming, GUO Ling-ling. Flight Simulator Overview [J]. Silicon Valley,2010(14):140-140.

[10] YANG Hao-quan, ZHAO Ke-ding, WU Sheng-lin.Key Technologies and Developing State of 6-DOF Motion System for Flight Simulator[J]. JOURNAL OF SYSTEM SIMULATION, 2002,14(1):84-87.

[11] SHENG Xiao-wei. Research of Technologies of Sound Analysisi/Syntesis and Simulation for Flight SIMULATOR [D]. Harbin Institute of Technology,2012.

[12] ZHANG Yan-yan, HUANG Qi-tao, HAN Jun-wei. Desigh and Implementation of Visual

Simulation System in Flight Simulator[J].Journal of System Simulation,2009,21(12):3662-3667.

[13] GAO Zhen-xing. Research on Real - time Simulation Modeling of Large - scale Aircraft Flight under Complex Atmospheric Disturbance [D]. Nanjing University of Aeronautics and Astronautics,2009.

[14] ZHOU Qiang, LUO Zhi-qiang, LI Qiao.On Reliability Model of SCI Interconnections in Avionics System[J]Journal of Beijing University of Aeronautics and Astronautics,2001,27(5):536-539.

[15] ZHOU Tian-ran, SONG Li-ru, XIONG Hua-gang. Modeling and simulation of fibre channel foravionics[J]. ]Journal of Beijing University of Aeronautics and Astronautics,2008,34(10):1117-1120.

[16] GUO Xiu-yun, LI Hui-qin, LIANG Jian-ming. Introduction to Modeling Methods of Electromechanical System[J]. Mechanical and Electrical Engineering Technology,2005,34(12):13-16.

[17] WANG Li-wen, ZHANG Lei, WEI Ming-kun. Research and Implementation of a Sound Simulation System for Flight Simulation Based on Direction[J]. Hydraulic And Pneumatic,2009(6):51-54.

[18] LIU Yan, YANG Qing-hua. Desigh on Airborne ARING429 Bus Test System Based on LabVIEW[J]. Manufacturing Automation,2015(11):10-12.

[19] ZHANG Xiao-fei. Modeling and Simulation of the Flight Vehicle Dynamics for Motion Simulation[D]. Beijing Jiaotong University ,2014.

[20] YANG Yv-lei. Research on Models of Automatic Flight System of Civil Aircraft[D]. Nanjing University of Aeronautics and Astronautics,2012.

[21] QV Xiao-yu. Preliminary Research on Flight Simulation System[J]. Software Guide, 2014(9):95-96. 